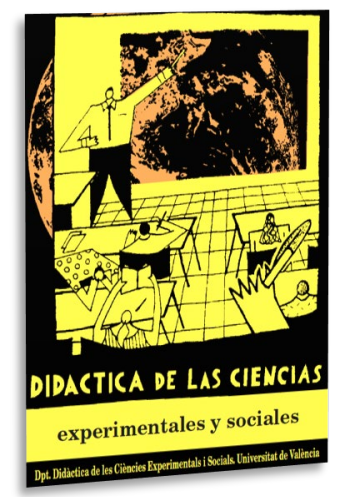

\title{
Identificación del alumnado de Educación Primaria con las narrativas históricas locales y nacionales. Estudio de caso en un contexto multicultural
}

\author{
Identification of Primary Education students \\ with local and national historical narratives. \\ Case study in a multicultural context
}

DOI:10.7203/DCES.38.13491

\author{
Nerea Cardoso Guillamón \\ Universidad de Murcia, nerea.cardoso@um.es \\ Sebastián Molina Puche \\ Universidad de Murcia, smolina@um.es \\ ORCID iD: https://orcid.org/0000-0003-1469-2100 \\ Jorge Ortuño Molina \\ Universidad de Murcia, jortunom@um.es \\ ORCID iD: https://orcid.org/0000-0002-4665-4910
}

\begin{abstract}
RESUMEN: En el presente trabajo se analiza la relación existente entre la enseñanza de la historia y la formación de identidades colectivas en una sociedad como la actual, caracterizada por la pluralidad cultural. Para ello, se ha realizado una investigación de carácter principalmente cualitativo en la que, mediante el empleo de cuatro instrumentos de recogida de información y de la triangulación de datos, se ha podido comprobar la necesidad de reformulación de los contenidos históricos por la falta de representación de la diversidad cultural en la escuela. A pesar de esto, los resultados nos han mostrado que el empleo de metodologías alternativas que fomenten la investigación, el trabajo cooperativo y el pensamiento crítico puede resultar una herramienta muy útil para superar las narrativas históricas presentes en el currículo de Educación Primaria.
\end{abstract}

Palabras Clave: educación histórica, identidad, Educación Primaria, pluralidad cultural, sentimiento de pertenencia.

ABSTRACT: This work analyses the relationship between the teaching of history and the formation of collective identities in the current society, which is characterized by cultural plurality. For this purpose, mainly qualitative research has been carried out with four data collection instruments and data triangulation which have allowed to verify the need to reformulate the historical contents due to the lack of representation of cultural diversity at schools. Despite this, the results have shown that the use of alternative methodologies that encourage research, cooperative work and critical thinking can be very useful to overcome the traditionally taught historical narratives present in the Primary Education curriculum.

KEYWORDS: historical education, identity, Primary Education, cultural plurality, sense of belonging.

Fecha de recepción: noviembre de 2018

Fecha de aceptación: abril de 2019

Este artículo es fruto del proyecto de investigación "La evaluación de las competencias y el desarrollo de capacidades cognitivas sobre Historia en Educación Secundaria Obligatoria” (EDU2015-65621-C3-2-R), subvencionado por el Ministerio de Economía y Competitividad. 


\section{JUSTIFICACIÓN DEL ESTUDIO}

Tras el nacimiento de la historia como área independiente en la educación formal a finales del siglo XIX y, debido a la estructura social predominante en España en ese momento, la educación perseguía unos objetivos que poco tienen que ver con los que se proponen en la actualidad. La formación de ciudadanos patrióticos, con una identidad nacional firme y homogénea se convirtió en la clave de esta materia, es decir, la educación histórica estaba destinada a informar, más que a formar, de los acontecimientos más relevantes que han tenido lugar en cada época histórica, haciendo hincapié en los datos, fechas y personajes más importantes en su desarrollo, desde un punto de vista centrado en el contexto de nuestro país y los propios intereses de aquellos que decidían los contenidos que debían ser impartidos. Siguiendo la propuesta de Giroux (1994), las escuelas se presentaban como esferas ideológicas y políticas en las que, por lo general, la cultura dominante intenta producir conocimientos y subjetividades que estén de acuerdo con sus propios intereses.

La pertinencia de este trabajo radica, precisamente, en la necesidad de analizar si la enseñanza de la historia en el ámbito de la educación formal permite o no dar respuesta a las realidades actuales o si, por el contrario, sigue cumpliendo la función de elemento de homogeneización nacional. Todo esto teniendo en cuenta no solo los contenidos a impartir, sino los métodos y las estrategias con que estos son impartidos por parte de los docentes y las posibles repercusiones que pueda tener la metodología empleada en la significación del aprendizaje de la historia en Educación Primaria. Lo que nos ayudará a entender cómo se construye la visión del pasado del alumnado y si esta visión es lo suficientemente crítica y multivocal, mostrando los principales elementos que forman en la actualidad las sociedades, que son tan dinámicos, híbridos y cambiantes.

\subsection{Identidad colectiva e historia}

La mezcla de culturas, costumbres, opiniones y maneras de entender la vida derivada de los cambios socioculturales experimentados en España durante los últimos años han tenido evidentes repercusiones en la formación de la propia identidad de las personas. Molina, Miralles, Deusdad y Alfageme (2017) afirman que la enseñanza de la historia y la creación de identidades (principalmente nacionales) han estado totalmente ligadas, y en buena parte siguen estándolo. En este sentido, la historia escolar representa una materia determinante en la configuración de identidades colectivas, especialmente en la etapa de Primaria por realizarse durante los años más importantes en el desarrollo social y emocional de los alumnos. En este sentido,

convendría, entonces, conferir a la enseñanza no solo de la historia, sino de las ciencias sociales, una orientación transcultural en respaldo al conocimiento de la diversidad humana, con un enfoque que sea más inclusivo y que supere la confrontación 'nosotros-ellos' (Aranguren, 2006: $6)$.

El cambio en la concepción de la identidad nacional se presenta en este momento como completamente necesario para asegurar una inclusión en todos los ámbitos de todos y cada uno de los alumnos con los que contamos en las escuelas, y no solo con aquellos que puedan sentirse representados por los valores tradicionales. La identidad patriótica que ha caracterizado siempre nuestro país se encuentra en un momento de fragilidad, y va dejando paso poco a poco a una identidad mucho más abierta, que debe favorecer la representación de todos. Como afirma Silva, "en cuanto tengamos sociedades que puedan expresarse a través de sus conflictos sin negar a los otros, sin silenciarlos, podremos estar seguros de que estas sociedades han abierto el horizonte de significación democrática, permitiéndose pensar desde la utopía y afirmando la posibilidad de interpretar el mundo como pluralidad de perspectivas" (2011: 61). 
La educación se convierte en la institución más adecuada para empezar a desarrollar ese cambio pues, tal y como afirma Carretero (2000), los contenidos de la historia escolar tienen una enorme fuerza en el proceso de conformación del conjunto de ideas y representaciones que forman la identidad nacional, una identidad que precisa ser cambiada y adaptada a las características de la población actual, por la necesidad de representar a todos los ciudadanos en su pleno derecho de pertenecer a la misma, y no para seguir viéndolos como extranjeros a los que culpar de los desastres del pasado. De este modo, como afirman Molina, Miralles y Ortuño (2013), se podrá entender el estrecho vínculo que existe entre la formación en historia del alumnado y el desarrollo de una ciudadanía crítica, responsable y democrática.

En un momento como en el que vivimos en el que la inmediatez, la información y la comunicación se han convertido en sus pilares fundamentales, resulta imprescindible contar con mecanismos que nos ayuden a comprendernos a nosotros mismos, a valorar, juzgar e integrar en nuestras vidas todas aquellas prácticas que nos definan, pues todos necesitamos tener nuestra propia identidad, siendo para Pérez Garzón (2002) una de las necesidades humanas más universales.

Esta sociedad en la que los rasgos de culturas que han estado a lo largo del tiempo separadas por barreras, principalmente geográficas, hoy en día se mezclan para formar una sociedad donde los marcos de referencia están compuestos por elementos culturales diversos, de forma que los límites culturales tradicionales de la sociedad ahora ya no son tan definidos, si es que alguna vez lo fueron. Cada individuo se mueve en una sociedad interconectada, siendo esa interconexión un elemento más de la identidad y de la definición de la nueva forma de socializarse, que se caracteriza por la creación de redes, tanto en el plano físico como virutal, y que complejiza y dinamiza la interacción. Así, los individuos eligen las redes de comunicación y socialización, dando lugar a una sociedad hipersocial, no de individuos aislados, y tremendamente diversa (Castells, 2005). La presencia cada vez mayor de esta diversidad en nuestras aulas requiere, como veremos en el apartado siguiente, la aceptación y el reconocimiento de la pluralidad como medio de enriquecimiento e integración de toda la ciudadanía a la sociedad.

En este momento de nuestro discurso comprendemos la necesidad de seguir trabajando acerca de la inclusión, por la necesidad que sigue habiendo hoy en día de defenderla como un derecho y dejar de verla como un favor. Como afirma Aranguren (2006), el interculturalismo del ámbito educativo necesita una nueva conceptualización basada en fundamentos que aboguen por la integración de la diversidad, el pluralismo y las identidades múltiples.

\subsection{Educación histórica para una sociedad inclusiva en el siglo XXI}

Del mismo modo que definió como dura la sociedad del siglo XIX, Zygmunt Bauman (1996) acuñó, de manera bastante acertada, el término "líquido" para definir la sociedad en la que nos encontramos hoy en día. La modernidad líquida a la que él se refiere representa la fragilidad de los valores que han sustentado a la sociedad a lo largo del tiempo, es decir, la presencia de valores basados en el nacionalismo y el patriotismo y la diferencia del "nosotros" y el "ellos" para referirnos a todas aquellas personas ajenas a nuestra sociedad, cultura y costumbres. Personas que han sido tradicionalmente vistas desde fuera y que hoy en día forman una parte indisoluble de nuestra sociedad, no solo de manera geográfica, sino también identitaria y social. Por ello, tal y como afirma Cuesta (2000), los cambios sociales y culturales acaecidos en los últimos veinte años han producido una crisis de identidad del currículum tradicional de Historia y de las funciones educativas que se imputan al conocimiento histórico.

Desde el punto de vista de Bauman, debemos comprender que nos encontramos en una época de pluralismo cultural, y no de pluralidad de culturas (1999), perdiendo, consecuentemente, todo su sentido en la sociedad actual el concepto de nación que se había inculcado hasta este momento, principalmente desde el área de Historia de España. "A la vista de tales diferencias cabe preguntarse 
si un aprendizaje común en materia de historia y, a la postre, de política, tiene (todavía) una justificación pedagógica y una aplicación posible a la práctica” (Von Borries, 2006: 1).

Mejía y Mejía (2015) afirman que el principal problema de la enseñanza de la Historia es que ha estado tradicionalmente centrada en un enfoque transmisionista e historicista y configurada por unos contenidos completamente ajenos a la realidad de los estudiantes, su entorno y sus comunidades. Pero la historia tiene la capacidad de facilitar la inclusión de nuestro alumnado y, al mismo tiempo, de provocar que las diferencias entre unos y otros sigan aumentando, dependiendo de la forma en que esta sea contada, los contenidos que mostremos a nuestros alumnos y, sobre todo, la actitud con que lo hagamos.

Defender la posición de los alumnos como los sujetos más importantes de la educación supone tenerlos en consideración de manera integral, comenzando por la selección de los contenidos a impartir en cada área, siendo necesario que se sientan completamente representados en todos los procesos que se llevan a cabo en ellas. La escuela, lejos de servir de reflejo de esta nueva sociedad globalizada y heterogénea, sigue acentuando las diferencias entre los alumnos, provocando que se alejen cada vez más entre sí (Fontana, 2006).

Es preciso preguntarse en este momento por el objetivo final de la enseñanza de la historia en la actualidad. Son numerosos los autores que apuestan por el desarrollo del pensamiento histórico crítico en contraposición a las narrativas maestras que la han caracterizado desde sus inicios. Carretero, Castorina, Sarti, Alphen y Barreiro (2013), en este sentido, plantean la siguiente pregunta: ¿se puede amar al propio país y, al mismo tiempo desarrollar una comprensión crítica de su funcionamiento?

Como se ha expuesto en la justificación de este trabajo, la formación de ciudadanos críticos es uno de los objetivos de la educación. La Historia, lejos de ser una guía para lograrlo, puede suponer trabas y contraargumentos a este fin que dificultan enormemente su adquisición. Debemos huir, por tanto, de esta linealidad que ha caracterizado los discursos históricos y mostrar más la multicausalidad y la multivocalidad en ellos, mostrar la presencia de muchos más elementos en la historia de los que las narrativas maestras suelen contar. Porque el reto de la educación no está en enseñar más contenidos históricos, sino en entender que la historia se puede analizar de forma crítica, que no debe interesarnos solo lo que pasó, sino para qué algunas de esas historias pueden servirnos en la actualidad. De este modo lo afirma Fontana:

Pienso en una clase de historia que no aspire tanto a acumular conocimientos como a enseñar a pensar, a dudar, a conseguir que nuestros alumnos no acepten los hechos que contienen los libros de historia como si fuesen datos que hay que memorizar, certezas como las que se enseñan en el estudio de las matemáticas, sino como opiniones e interpretaciones que se pueden y se deben analizar y discutir (2006: 9).

Según la propuesta del National Council for the Social Studies de Estados Unidos de América de 1994 (citado en Pagès 2002: 258-259), el currículo de historia para la formación de ciudadanos y ciudadanas del siglo XXI debe ser: significativo, integrador, basado en valores, exigente intelectualmente y activo. De este modo, se conseguirá que cumpla con su propósito, como afirman Molina, Miralles, Deusdad y Alfageme:

La historia, además de una herramienta para tomar conciencia de la situación social del presente a través del análisis crítico del pasado, se convierte también en un instrumento para transformar la realidad, capacitar al alumnado como ciudadano crítico con su tiempo y capaz de comprometerse con su sociedad y actuar para la mejora de esta (2017: 335). 


\section{MARCO EMPÍRICO. METODOLOGÍA E INSTRUMENTOS DE RECOGIDA DE INFORMACIÓN}

\subsection{Problema y objetivo de la investigación}

La persistencia de la enseñanza de la historia como un elemento de aculturación y con escasa identificación de los colectivos sin voz tradicional en los discursos históricos es un problema serio para una sociedad en continuo cambio y dinamismo. Por ello, nos preguntamos si contribuye la enseñanza de la historia a la integración de todo el alumnado por medio de las narrativas que conforman la materia facilitando la creación de identidades colectivas mediante las que todo el alumnado se sienta identificado.

Para ello, se ha propuesto el siguiente objetivo general: Conocer la repercusión de la enseñanza de la historia en la creación de un sentimiento de pertenencia a la sociedad actual del alumnado de origen inmigrante en Educación Primaria. El cual se divide en los siguientes específicos:

- Analizar la adecuación de los contenidos del área a las características de la realidad pluriculatural del aula.

- Conocer la percepción docente acerca de la materia, la metodología que utilizan y sus posibilidades para favorecer la inclusión del alumnado.

- Comprobar la percepción y el interés del alumnado por los contenidos que conforman la materia y su contribución al desarrollo de valores de aceptación, tolerancia e inclusión social.

\subsection{Selección del caso}

La muestra seleccionada corresponde a los alumnos y alumnas de quinto curso de primaria de un centro público de localidad murciana de Cartagena, cuya diversidad cultural refleja perfectamente la pluralidad que caracteriza a nuestra sociedad en la actualidad. Aunque el aula objeto de estudio cuenta con un total de 20 alumnos, se seleccionó a la mitad de ellos con el objetivo de poder llevar a cabo una investigación mucho más exhaustiva de cada uno de ellos. Contamos con la colaboración, además, de cuatro maestros del centro: su tutora, el maestro de educación compensatoria la maestra de audición y lenguaje, y la jefa de estudios del centro (Tabla 1).

TABLA 1. Muestra seleccionada

\begin{tabular}{|c|c|c|c|c|c|c|}
\hline Profesor & Código & Sexo & Edad & Experiencia & $\begin{array}{l}\text { Años en } \\
\text { el centro }\end{array}$ & Formación Académica \\
\hline Tutora & P1 & $\mathrm{F}$ & 33 & 10 & 2 & $\begin{array}{l}\text { Educación Primaria } \\
\text { (Ed. Musical) }\end{array}$ \\
\hline Compensatoria & $\mathrm{P} 2$ & M & 26 & 2 & 2 & Educación Primaria \\
\hline $\begin{array}{l}\text { Jefa de } \\
\text { estudios }\end{array}$ & P3 & $\mathrm{F}$ & 34 & 11 & 5 & $\begin{array}{ll}- & \text { Educación Primaria } \\
\text { - } & \text { Pedagogía }\end{array}$ \\
\hline $\begin{array}{l}\text { Audición y } \\
\text { Lenguaje }\end{array}$ & P4 & $\mathrm{F}$ & 52 & 15 & 7 & $\begin{array}{ll}\text { - } & \begin{array}{l}\text { Educación Primaria } \\
\text { (Audición y Lenguaje) }\end{array}\end{array}$ \\
\hline
\end{tabular}

Fuente: elaboración propia

Por su parte, el perfil del alumnado trabajado corresponde a un barrio situado a unos 3 kilómetros al norte de Cartagena, reflejo perfecto de la diversidad cultural y social objeto de estudio en el presente trabajo. El centro escolar era de carácter público, situado en una de las zonas más pobres de la ciudad de Cartagena, rodeado de viviendas de gran antigüedad y bajo coste, lo que ha propiciado su ocupación por parte de las familias con menos recursos, siendo estas principalmente familias de origen inmigrante y de etnia gitana. La mayor parte del alumnado que acude a este centro es aproximadamente el $80 \%$ inmigrantes o de origen inmigrante, y prácticamente el $20 \%$ restante 
pertenece a minorías culturales, como la gitana. Los participantes en este estudio han sido 7 estudiantes de nacionalidad o progenitores de nacionalidad marroquí, uno ecuatoriano, otro guineano y otro de etnia gitana.

\subsection{Metodología de la investigación}

Debido al interrogante y a los objetivos planteados, la investigación cualitativa se presenta como el método más adecuado para la respuesta y consecución de los mismos. Dentro de esta metodología encontramos numerosas técnicas y métodos pero, dada la concreción del contexto en el que esta se lleva a cabo, se ha considerado el estudio de casos como el más adecuado para su desarrollo. Martínez Carazo lo define como una herramienta valiosa de investigación, y su mayor fortaleza radica en que a través del mismo se mide y registra la conducta de las personas involucradas en el fenómeno estudiado, pudiendo obtener sus datos desde una variedad de fuentes, tanto cualitativas como cuantitativas (2006: 167).

El estudio de casos concretos requiere una recogida de información muy exhaustiva que permita conocer el máximo de los sujetos sobre los que basamos la investigación con el fin de obtener toda la información necesaria y poder establecer unas conclusiones realmente significativas. Por este motivo, nos valemos de la triangulación para realizarla. Okuda y Gómez-Restrepo (2005: 119) la definen como el uso de varios métodos de fuentes de datos, de teorías, de investigadores o de ambientes en el estudio de un fenómeno. De este modo, disminuye la posibilidad de malentendidos, mediante la producción de información redundante que permite esclarecer los significados y verificar la repetibilidad de una observación, aumentando la fiabilidad de los resultados.

\subsection{Técnicas de recogida de datos}

Atendiendo a la metodología empleada, se diseñaron cuatro instrumentos de recogida de datos que permitieron contrastar, por medio de diversas fuentes, las aportaciones de todos los sujetos participantes en la investigación. La siguiente tabla (Tabla 2) muestra los diferentes instrumentos utilizados y los sujetos con los que se llevaron a cabo.

TABLA 2. Instrumentos de investigación utilizados

\begin{tabular}{|cc|}
\hline Instrumentos & Participantes \\
\hline Observación directa (OD) & Alumnado \\
\hline $\begin{array}{c}\text { Entrevista semi-estructurada } \\
\text { (EP) }\end{array}$ & Profesorado \\
\hline $\begin{array}{c}\text { Cuestionario de escala Likert } \\
\text { (CA) }\end{array}$ & Alumnado \\
\hline Actividad de aula (AA) & Alumnado \\
\hline \multicolumn{2}{|c|}{ Fuente: elaboración propia } \\
\hline
\end{tabular}

Para asegurar la triangulación de todos ellos, se establecieron ocho categorías de análisis para los diferentes objetivos específicos del estudio, relacionando estas con cada uno de los instrumentos utilizados por medio de los ítems que conforman cada uno de ellos. La relación entre los objetivos, las categorías de estudio y los instrumentos utilizados para cada una se muestra en la Tabla 3. 
TABLA 3. Relación objetivos-categorías-instrumentos de investigación.

\begin{tabular}{|c|c|c|}
\hline \multirow{4}{*}{$\begin{array}{l}\text { OBJETIVOS ESPECÍFICOS } \\
\text { 1. Analizar la adecuación de los } \\
\text { contenidos del área al momento } \\
\text { histórico y las características de la } \\
\text { sociedad actual. }\end{array}$} & CATEGORÍAS & INSTRUMENTOS \\
\hline & $\begin{array}{l}\text { Contexto del centro y relaciones } \\
\text { personales. }\end{array}$ & $\mathrm{OD} / \mathrm{EP} / \mathrm{CA}$ \\
\hline & $\begin{array}{l}\text { Objetivos de la historia a nivel } \\
\text { curricular. }\end{array}$ & $\mathrm{OD} / \mathrm{EP} / \mathrm{CA}$ \\
\hline & $\begin{array}{l}\text { Valores desarrollados por medio de los } \\
\text { contenidos. }\end{array}$ & $\mathrm{OD} / \mathrm{EP} / \mathrm{CA}$ \\
\hline $\begin{array}{l}\text { 2. Conocer la percepción docente } \\
\text { acerca de la metodología empleada y }\end{array}$ & $\begin{array}{l}\text { Repercusión de la metodología } \\
\text { empleada. }\end{array}$ & $\mathrm{OD} / \mathrm{EP} / \mathrm{CA}$ \\
\hline $\begin{array}{l}\text { sus posibilidades para favorecer la } \\
\text { inclusión del alumnado. }\end{array}$ & $\begin{array}{l}\text { Conciencia docente acerca de la } \\
\text { adecuación de los contenidos. }\end{array}$ & $\mathrm{EP} / \mathrm{CA}$ \\
\hline 3. Comprobar la percepción y el & Interés por la materia y sus contenidos. & $\mathrm{OD} / \mathrm{EP} / \mathrm{CA} / \mathrm{AA}$ \\
\hline interés del alumnado por los & Relación entre la historia y la actualidad. & $\mathrm{EP} / \mathrm{CA}$ \\
\hline $\begin{array}{l}\text { contenidos que conforman la materia y } \\
\text { su contribución al desarrollo de } \\
\text { valores de aceptación, tolerancia e } \\
\text { inclusión. }\end{array}$ & Implicación, tolerancia e inclusión. & $\mathrm{OD} / \mathrm{CA}$ \\
\hline
\end{tabular}

Fuente: elaboración propia

\section{Presentación de Resultados}

\subsection{Objetivo 1: adecuación de los contenidos de historia a la sociedad actual.}

\section{- Categoría A: contexto del centro y relaciones personales}

La primera categoría mide las relaciones personales que existen entre el alumnado y la percepción de los maestros acerca de sus interacciones, además de la identificación del alumnado a nivel personal con los contenidos que aprenden (Tabla 4).

TABLA 4. Categoría contexto del centro y relaciones personales: ejemplos de respuestas.

\begin{tabular}{|c|c|c|}
\hline \multicolumn{3}{|c|}{ CONTEXTO DEL CENTRO Y RELACIONES PERSONALES } \\
\hline \multicolumn{3}{|c|}{$\begin{array}{l}\text { 3. ¿Qué relación mantienen los alumnos y alumnas entre sí?, ¿crees que existe algún tipo de } \\
\text { discriminación en el aula? }\end{array}$} \\
\hline & Profesor & Respuesta \\
\hline \multirow[t]{2}{*}{$\begin{array}{l}\text { No hay } \\
\text { discriminación }\end{array}$} & P1 & $\begin{array}{l}\text { El año pasado, cuando yo llegué no había mucha discriminación, } \\
\text { pero no se conocían a pesar de que llevaban juntos desde los tres } \\
\text { años. Estos dos años que hemos estado juntos se han convertido en } \\
\text { un grupo (...) }\end{array}$ \\
\hline & $\mathrm{P} 2$ & $\begin{array}{l}\text { Yo creo que, en líneas generales, tienen una buena relación. Son } \\
\text { como una pequeña familia entre ellos, aceptan la diversidad que hay } \\
\text { entre los diferentes miembros de las clases y del centro }(\ldots)\end{array}$ \\
\hline \multirow[t]{2}{*}{$\begin{array}{l}\text { Hay discriminación } \\
\text { por factores externos }\end{array}$} & P3 & $\begin{array}{l}\text { La relación entre ellos es bastante normalizada, de hecho, no es la } \\
\text { relación entre los alumnos la que me preocupa, es la influencia de los } \\
\text { padres. En el centro no hay discriminación por diferencias culturales } \\
\text { o étnicas, pero sí hay casos en los que en casa escuchan comentarios } \\
\text { que son discriminatorios y se les pueden quedar. }\end{array}$ \\
\hline & P4 & $\begin{array}{l}\text { Yo creo que entre ellos cuando se olvidan de las cosas no hay } \\
\text { discriminación, pero muchas veces viene de casa. Y muchas veces el } \\
\text { idioma hace que se junten para jugar unos con otros. }\end{array}$ \\
\hline
\end{tabular}


En el ítem derivado de las rúbricas de observación directa comprobamos que el $60 \%$ de los alumnos participantes valoran a todos sus compañeros por igual, frente a un $40 \%$ que no lo hace o lo hace a veces. La percepción de los maestros en cuanto a las relaciones del alumnado no es demasiado diferente. Aunque todos ellos coinciden en que no hay discriminación entre el alumnado, dos de ellos puntualizaron que a veces se realizan comentarios ofensivos valiéndose de las diferencias culturales entre ellos. En cuanto a la pregunta de la entrevista, todos ellos coinciden en que el trabajo en este centro, a pesar de sus dificultades, es muy gratificante.

De los ítems analizados del cuestionario del alumnado, ambos relacionados con el tratamiento de las culturas en la escuela concluimos que, a pesar de que el $90 \%$ reconoce el estudio de diversas culturas, solo el $60 \%$ se encuentra reconocido en estos discursos.

\section{- Categoría B: objetivos de la historia a nivel curricular}

La segunda categoría fue establecida para comprobar los objetivos de la historia desde el currículum. Los ítems pertenecientes a la rúbrica de observación directa relacionados con la adquisición de los contenidos de la materia por parte del alumnado muestran una falta de conocimientos previos, de utilización de vocabulario adecuado y de seguridad a la hora de exponerlos por parte de más del $40 \%$ del alumnado, mientras que solo el $30 \%$ demuestra dominar estos contenidos de manera habitual.

En cuanto al cuestionario del alumnado, las preguntas relacionadas con la utilidad de la historia en la vida cotidiana del alumnado obtuvieron resultados bastante positivos, admitiendo esta utilidad un $80 \%$ y un $70 \%$ del alumnado. Sin embargo, si contrastamos estas respuestas con las aportadas por los docentes en su entrevista, comprobamos que la percepción de todos ellos es contraria, admitiendo que los objetivos siguen orientados al aprendizaje memorístico de contenidos completamente descontextualizados del presente.

\section{- Categoría C: valores desarrollados por medio de los contenidos}

La última categoría estaba dedicada a comprobar la adquisición de valores por medio de los contenidos históricos. La perspectiva del alumnado mediante sus respuestas a ambas preguntas del cuestionario es que los contenidos de la materia les ayudan a desarrollar valores de tolerancia hacia el resto de sus compañeros por el modo de tratar todas las culturas estudiadas. Al igual que en la categoría anterior, es el $80 \%$ del alumnado quien lo afirma. Esto lo podemos confirmar con los datos aportados por la observación directa al comprobar que la mayoría de ellos intenta crear un clima de aula relajado, y el 50\% participa de manera activa en la resolución de los conflictos que surgen en el aula.

Aunque los datos anteriores son bastante positivos, la percepción docente muestra lo contrario. La mitad de los maestros entrevistados opina que los contenidos de la historia siguen estando demasiado orientados al desarrollo de valores patrióticos y nacionalistas, lo que dificulta la enseñanza de una historia representativa para todo el alumnado y que sirva, además, como medio para fomentar valores positivos con los demás. El resto de los maestros opina que, no solo los contenidos, sino ellos mismos son los que tienen la mayor capacidad para guiar estos hacia la inclusión, dependiendo de la metodología que apliquen y, en ocasiones, de sus propios intereses personales.

\subsection{Objetivo 2: percepción docente acerca de la metodología empleada}

\section{- Categoría D: Repercusión de la metodología empleada}

Como apuntábamos en la categoría anterior, la metodología docente es uno de los factores que pueden influir en el alumnado. En primer lugar, debemos destacar que el centro en el que se ha centrado esta investigación utiliza, de manera general, el trabajo por proyectos como metodología de 
enseñanza (Tabla 5). El porqué de la utilización de la misma lo encontramos en las respuestas de los maestros a la cuarta pregunta de su entrevista. Todos ellos defienden el trabajo por proyectos como un método eficaz, no solo por la adquisición de aprendizajes significativos para el alumnado, sino porque aporta beneficios en las relaciones sociales entre el grupo de alumnos. Tener que trabajar en grupo, investigar, crear los contenidos y mostrarlos al resto de compañeros hace que mejore su comunicación, el respeto por los demás y la creación de un grupo mucho más cohesionado. En palabras de una de las maestras entrevistadas (P4), el trabajo por proyectos "les hace unirse, colaborar, participar, les hace creerse parte de un grupo".

TABLA 5. Repercusión de la metodología empleada en el aula de historia: ejemplos de respuestas

\begin{tabular}{|c|c|c|}
\hline \multirow{2}{*}{\multicolumn{3}{|c|}{$\begin{array}{l}\text { REPERCUSIÓN DE LA METODOLOGÍA EMPLEADA } \\
\text { odología docente utilizas normalmente en tus clases?, ¿crees que el uso de } \\
\text { is participativas influye de manera positiva en sus relaciones personales? }\end{array}$}} \\
\hline & & \\
\hline & Profesor & Respuesta \\
\hline \multirow[t]{4}{*}{ Metodologías activas } & P1 & $\begin{array}{l}\text { La metodología influye en que esas relaciones sean así, mucho. La } \\
\text { metodología en sí no es una específica, utilizo lo mejor o lo que más } \\
\text { me gusta de cada una de las metodologías que voy aprendiendo. } \\
\text { Sobre todo, utilizo metodologías manipulativas, activas, que hablen, } \\
\text { que se muevan, grupos cooperativos, investigaciones, etc. }\end{array}$ \\
\hline & $\mathrm{P} 2$ & $\begin{array}{l}\text { La metodología influe en la creación de esa piña, yo creo que sí, } \\
\text { porque al trabajar por proyectos, como se intentan trabajar la mayoría } \\
\text { de los contenidos, es verdad que tienen mucho trabajo creativo en } \\
\text { grupo, y que al final entre todos tienen que ir alcanzando un objetivo } \\
\text { común, que no es cada uno a lo suyo, su aprendizaje y sin importar } \\
\text { lo del compañero (...) }\end{array}$ \\
\hline & P3 & $\begin{array}{l}\text { Yo imparto principalmente matemáticas, y en mis clases utilizo el } \\
\text { método ABN. En general, intento trabajar mucho las habilidades } \\
\text { sociales, utilizar mucho los trabajos cooperativos, las exposiciones, } \\
\text { hacer que se expresen, todo lo que pueda hacer que aprendan a } \\
\text { comunicarse entre ellos. } \\
\text { También intento trabajar mucho las normas y que sean clases } \\
\text { realmente democráticas. Es muy bonito decir que una clase es } \\
\text { democrática pero no se suele demostrar. Yo intento hacer que tengan } \\
\text { que tomar decisiones de verdad, que se escuchen y lleguen a } \\
\text { acuerdos, al tomar cualquier tipo de decisión en el aula, eso también } \\
\text { les ayuda mucho en sus relaciones. }\end{array}$ \\
\hline & P4 & $\begin{array}{l}\text { Los tutores son vitales. He visto pasar muchos tutores por el centro, } \\
\text { porque yo entro en todas las clases (desde } 3 \text { años hasta sexto), por lo } \\
\text { que veo como trabajan todos los tutores. Lo que más me ha gustado } \\
\text { ha sido el trabajo por proyectos; les hace unirse, colaborar, participar, } \\
\text { les hace creerse parte de un grupo, y esto influye directamente en los } \\
\text { alumnos. }\end{array}$ \\
\hline
\end{tabular}

Si analizamos las respuestas aportadas por el alumnado comprobamos cómo su opinión acerca de esta metodología no dista de la de los maestros. El 70\% de ellos admiten que el aprendizaje basado en proyectos no es solo un método más divertido que el tradicional, sino que con él aprenden más al tener que involucrarse en todo el proceso.

Los datos recogidos por la observación directa, aunque con excepciones, no hacen más que confirmar lo aportado por las fuentes anteriores. Durante la observación de los proyectos se comprobó cómo la implicación del alumnado en el proceso era elevada, interesándose la mayoría de ellos por la información que debían buscar, seleccionando la más interesante y redactándola para compartirla con 
el resto de sus compañeros y utilizando los medios electrónicos de manera adecuada tanto en la búsqueda de información como en las exposiciones posteriores.

\section{- Categoría E: conciencia docente acerca de la adecuación de los contenidos}

La siguiente categoría fue creada con la intención de estudiar la percepción de los maestros acerca de la adecuación de los contenidos de Historia teniendo en cuenta las características de la sociedad en general y de este centro en particular.

La primera pregunta de la entrevista, relativa a la importancia del aprendizaje de la historia en Educación Primaria, nos muestra muchas similitudes entre las opiniones del profesorado. Todos ellos coinciden en la importancia de esta materia por sus posibilidades para crear en el alumnado un sentimiento de pertenencia al país al contar el transcurso de la historia y de todos los pueblos que han formado parte del mismo. En este sentido, la consideran especialmente necesaria para el alumnado por la falta de información que reciben del exterior debido al desconocimiento de esta historia por parte de sus familias. Uno de los docentes entrevistados apunta lo siguiente: "son temas que yo creo que en su casa no se tratan, ni ven las noticias, etc., entonces yo creo que es la forma de que ellos conozcan su propia procedencia."

TABLA 6. Conciencia docente acerca de la adecuación de los contenidos: ejemplos de respuestas

\section{CONCIENCIA DOCENTE ACERCA DE LA REPERCUSIÓN DE LOS CONTENIDOS}

8. ¿Crees que la Historia que se enseña es la adecuada?

Profesor

La historia entendiéndola como acontecimientos pasados, sí que es verdad que se enseña lo que de verdad ha ocurrido, que a lo mejor se podría incidir más en otras partes, o incluso un enfoque de la historia

P2 como ¿qué se ha hecho para cambiar la historia?, no estudiar en este tema pasó esto, esto y esto, en esta época esto. La historia que se enseña es lineal, pero separando, a lo mejor debería centrarse más en las transiciones, en todo lo que afecta a la historia: la economía, la sociedad, la política, etc. Creo que deberían estar como un poco más globalizados los contenidos.

Se podría mejorar. A mí me gusta empezar por una cosa y no saber por dónde vas a acabar, y eso los estándares no lo permiten, y yo creo que

P4 falta un poco de cultura en general. No nos podemos limitar a una época en cada curso, sino saber un poquito de todo en todos los cursos y no llegar a sexto y entonces ver la actualidad y que se nos haya olvidado la Prehistoria, la Edad Media.

Ahí me pillas un poco porque no he dado mucha historia, solo he estado dos cursos con sociales y realmente no lo sé, a lo mejor hay mucho contenido, se debería ir poco a poco, por ejemplo, se meten las distintas etapas de golpe, a lo mejor la Prehistoria y la Edad Antigua, son etapas muy largas en las que se podría profundizar un poco más, no ir tan rápido. Este año hemos dado la Edad Media y la Edad Moderna, no da tiempo a verlo todo en profundidad.

Creo que los contenidos que se enseñan si son los adecuados, aunque todo se puede mejorar. La historia es una asignatura que requiere mucha memoria, es complicado, para conocerla tienes que conocer los datos y

P3 saber contextualizarlos, y para eso no queda otra opción que memorizarlos (...) las metodologías son las que empeoran o mejoran una materia. Creo que en esta materia en concreto la parte creativa y autónoma está más descuidada, pero porque la materia así lo requiere.

Fuente: elaboración propia 
En cuanto a la segunda pregunta, relativa a la percepción de los maestros acerca de la adecuación de los contenidos impartidos, encontramos diversidad de opiniones (Tabla 6). Dos de los maestros entrevistados (P2 y P4) consideran que los contenidos son adecuados, es decir, que la historia contada es la que ha de ser, y señalan como el principal problema que la materia se encuentra muy compartimentada en etapas, impidiendo enseñarla como una continuidad del tiempo. Los otros dos maestros (P1 y P3), consideran que los contenidos no son los adecuados, afirmando que estos están completamente descontextualizados, lo que dificulta que el alumnado comprenda la repercusión de todos los acontecimientos y sean capaces de comprender el presente. Encontramos en todos ellos un matiz, tal y como comentamos en la categoría referente a la metodología, pues todos afirman que el papel del maestro es fundamental, y que el desarrollo del pensamiento histórico crítico está más influenciado por el modo en el que se enseñe la materia que por los propios contenidos impartidos.

La última pregunta a los maestros pretendía comprobar hacía dónde están encaminados sus propios objetivos al enseñar la materia. Al igual que en la pregunta anterior, podemos dividir las respuestas de los docentes en dos categorías. Dos de los maestros (P3 y P4) consideran que su principal objetivo (al impartir cualquier materia) es que, tras impartir esos contenidos, el alumnado quiera saber más, que les guste lo que aprenden y, sobre todo, que les sirva para ser tolerantes. Los otros maestros (P1 y P2) orientan sus respuestas hacia la necesidad de conocer el pasado y, especialmente, el legado que han ido dejando a su paso las diferentes culturas para fomentar la inclusión del alumnado.

En relación con el desarrollo del pensamiento crítico en el alumnado se propuso una pregunta en el cuestionario para comprobar si a la hora de evaluar la materia los alumnos tenían libertad para expresar sus opiniones o se trataba siempre de preguntas conceptuales centradas en hechos puntuales. La opinión del alumnado nos muestra que sí pueden expresarlo, dándole el $70 \%$ de ellos la máxima puntuación en la escala. Las otras dos preguntas, más orientadas a la metodología, pretendían medir su opinión acerca de la implicación docente a la hora de realizar los proyectos y la variedad de fuentes que podían consultar. Ambas preguntas obtuvieron en un 90\% la máxima puntuación.

\subsection{Objetivo 3: percepción e interés de los alumnos por la materia}

\section{- Categoría F: interés por la materia y sus contenidos}

La siguiente categoría representa una de las centrales del proyecto, por ello, se dedicaron diez ítems a su estudio: cinco relacionados con la observación directa, dos pertenecientes a las entrevistas del profesorado, dos extraídos del cuestionario del alumnado y la actividad de aula completa.

Aproximadamente la mitad del alumnado observado mostraba interés tanto por sus temas como por los de sus compañeros, es decir, por la etapa histórica estudiada al completo. Sin embargo, solo el 30\% de los alumnos mostraba la motivación suficiente como para seguir investigando más allá de los contenidos mínimos propuestos. En cuanto a su actitud durante la realización del proyecto, la del $60 \%$ era bastante positiva, porcentaje compartido con el ítem relacionado con la claridad y seguridad durante la exposición. Si analizamos el cuestionario del alumnado, encontramos respuestas que confirman lo observado. El 50\% del alumnado afirma que los contenidos que aprenden son de su interés y solo el 10\% se muestra totalmente en contra. El mismo porcentaje es el obtenido al preguntar si recuerdan los contenidos estudiados con el paso del tiempo, aunque aumenta a un $20 \%$ el alumnado que niega recordarlos.

En cuanto a la primera pregunta de la entrevista analizada, relativa a las materias preferidas por el alumnado, tres de los maestros (P1, P2 y P3), coinciden en sus respuestas, afirmando que las asignaturas que prefieren son aquellas que se desarrollan normalmente fuera del aula de referencia por su metodología generalmente lúdica y participativa (Tabla 7). 
TABLA 7. Asignaturas preferidas por el alumnado: ejemplo de respuestas

\begin{tabular}{|c|c|c|}
\hline \multicolumn{3}{|c|}{$\begin{array}{l}\text { INTERÉS POR LA MATERIA Y SUS } \\
\text { CONTENIDOS }\end{array}$} \\
\hline \multicolumn{3}{|c|}{$\begin{array}{c}\text { Ordenación de las diferentes materias del curso } \\
\text { por preferencia (6 áreas en total) }\end{array}$} \\
\hline Alumno & Interés & Posición del área de Historia \\
\hline 1 & Bajo & $5^{\circ}$ posición \\
\hline 2 & Medio & $3^{\circ}$ posición \\
\hline 3 & Medio & $4^{\mathrm{o}}$ posición \\
\hline 4 & Bajo & $6^{\mathrm{o}}$ posición \\
\hline 5 & Bajo & $6^{\circ}$ posición \\
\hline 6 & Medio & $4^{\circ}$ posición \\
\hline 7 & bajo & $6^{\circ}$ posición \\
\hline 8 & Alto & $1^{\mathrm{o}}$ posición \\
\hline 9 & Alto & $2^{\mathrm{o}}$ posición \\
\hline 10 & Alto & $2^{\mathrm{o}}$ posición \\
\hline
\end{tabular}

Para averiguar las materias favoritas del alumnado, se realizó una actividad en el aula en la que debían ordenar seis de ellas por preferencia. Para evitar que las respuestas estuvieran influenciadas por la presencia del observador, la actividad fue realizada por la tutora durante una sesión de otra materia. Para el análisis de la actividad se crearon tres subcategorías para clasificar las respuestas del alumnado en función de su posicionamiento de la historia en la lista. De los diez alumnos participantes, solo tres de ellos consideran la de Historia como una de sus dos materias preferidas, al igual que en la segunda subcategoría, referente a un nivel medio de preferencia. Del resto del alumnado, el cual eligió la Historia como una de las dos materias que menos le gustan, tres de ellos la posicionaron en último lugar.

\section{- Categoría G: relación entre la Historia y la actualidad}

Para comprobar la opinión del alumnado acerca de la relación entre el estudio de la Historia y la actualidad se seleccionaron cuatro ítems objeto de estudio: una pregunta de la entrevista del profesorado y tres ítems del cuestionario del alumnado.

En el cuestionario del alumnado, encontramos en el primero de los ítems, en el que se preguntaba al alumnado por las consecuencias de los hechos pasados en el presente, que todos ellos afirmaron que tiene repercusiones. Sin embargo, cuando estudiamos las respuestas de los ítems 21 y 23, relacionados con la utilidad de la historia en relación con la comprensión del presente y el conocimiento de los hechos más relevantes del pasado, los porcentajes positivos disminuyen hasta un $40 \%$ y un $60 \%$, respectivamente.

\section{- Categoría H: implicación, tolerancia e inclusión}

La última categoría se creó con el fin de analizar las actitudes del alumnado, tanto con el resto de sus compañeros como con el propio proyecto, durante su realización y exposición, además de su motivación con los contenidos trabajados. Para ello, se seleccionaron nueve ítems, pertenecientes a la observación directa y al cuestionario.

El primero de ellos trataba de medir la actitud de cada alumno con los miembros de su grupo durante la búsqueda de información y la redacción del proyecto. Los resultados de este ítem nos muestran que un $70 \%$ del alumnado mantiene siempre una actitud positiva con los demás. Relacionado con este ítem se encuentra el $\mathrm{n}^{\circ}$. 13, en el que se medía si las aportaciones de los 
compañeros eran escuchadas con respeto y tolerancia por parte del resto. A pesar del alto porcentaje obtenido en el ítem anterior, en este disminuye hasta el 50\% el porcentaje de alumnado que valora y respeta el resto de las aportaciones siempre.

A continuación, encontramos cuatro ítems relacionados con la implicación de cada alumno, en los que se miden la capacidad de liderazgo en el proyecto de cada uno de ellos, el nivel de contribución a su desarrollo con respecto a los demás, la búsqueda del enriquecimiento del proyecto por medio de la creación de debates y su capacidad para mantener la atención de sus compañeros durante las exposiciones. Los resultados obtenidos para estos ítems son los siguientes: solo el $40 \%$ del alumnado muestra tanto interés como para ser un líder en su equipo, del 60\% restante, llama la atención que el $40 \%$ de ellos nunca hace nada para liderar a su equipo. En cuanto al nivel de contribución de cada uno, encontramos un 50\% del alumnado que se implica al máximo nivel, mientras que el mismo 40\% del ítem anterior esquiva participar de manera activa. Solo el 30\% del alumnado se involucra hasta el nivel de crear debates (comparando contenidos, haciendo conjeturas, etc.) que puedan aportar diferentes puntos de vista y enriquecer el proyecto, el 30\% de los alumnos restantes ni si quiera participa en ellos. Por último, relacionado con la capacidad para mantener la atención de sus compañeros durante la exposición, encontramos que el 50\% del alumnado lo consiguen debido a su seguridad al exponer y a su conocimiento de los contenidos, solo el $10 \%$ de ellos pierde toda la atención por no ser capaz de exponer con fluidez.

El último ítem analizado en esta categoría pertenece al cuestionario del alumnado y trata de medir si el aprendizaje de otras culturas, en el caso de los alumnos de origen inmigrante de su cultura de referencia, ayuda a su implicación en el aula y a su motivación por seguir aprendiendo. Los resultados muestran que el $80 \%$ del alumnado sí siente esa motivación al encontrar contenidos relacionados con su cultura, mientras que el $20 \%$ restante no encuentra en ellos ninguna motivación extra.

\section{DISCUSIÓN DE LOS RESULTADOS}

\subsection{Objetivo 1: adecuación de los contenidos de historia a la sociedad actual}

Se ha podido observar durante la investigación que las relaciones del alumnado están basadas en el respeto y la tolerancia hacia los demás, confirmando desde todos los instrumentos utilizados que no existe ningún tipo de discriminación. A pesar de esto, destaca un apunte común a las respuestas del cuerpo docente, admitiendo todos ellos que, en ocasiones en las que surge un problema en el aula, los alumnos hacen uso de comentarios racistas o discriminatorios para atacar a sus compañeros, y que estos comentarios son trasladados desde las familias en la mayoría de las ocasiones. Confirmamos, de este modo, que la influencia de las familias puede suponer un obstáculo al desarrollo de valores de respeto y tolerancia por medio de aportaciones contrarias a las que se pretende transmitir desde la escuela.

La educación es propuesta por Carretero y Kriger (2004) como el dispositivo primario de la socialización y, por tanto, requiere periódicas recontrataciones y renovaciones para adaptarse a las características de cada momento, favoreciendo que esta socialización se logre asegurando el reconocimiento de todos los que forman parte de ella. Aunque los objetivos de la educación en la actualidad están orientados, como se comentó en la justificación del trabajo, al desarrollo de prácticas inclusivas, de convivencia, conocimiento y reconocimiento de todos sus miembros, y se ha comprobado que el clima del aula es, generalmente, positivo y respetuoso, no se pueden achacar estos logros a los contenidos de la historia, de hecho, parecen ser más perjudiciales para su adquisición.

Aunque el alumnado, mediante sus respuestas al cuestionario, afirmó que el tratamiento de la diversidad cultural en los contenidos de la materia es suficiente, tanto el observador como los maestros entrevistados opinaron de manera contraria. La compartimentación de los contenidos, divididos en etapas históricas que se imparten de manera completamente aislada, la enseñanza basada 
en la memorización de fechas, batallas y hechos heroicos sin ninguna contextualización sobre sus causas o consecuencias, las continuas omisiones de los acontecimientos que no interesa resaltar, la enseñanza de otras culturas por medio únicamente de su folklore, impiden que el alumnado obtenga una visión global de la Historia que le permita juzgarla y utilizarla de manera significativa para comprender los hechos del presente o buscar justificaciones en el carácter dinámico e híbrido de las culturas que favorezcan la inclusión de la alteridad.

Por tanto, el desarrollo del pensamiento histórico crítico, considerado como una de las potencialidades de la enseñanza de la historia, en palabras de Mejía y Mejía (2015), por su capacidad para dotar al alumnado de los instrumentos necesarios para el análisis, la comprensión y la interpretación de los hechos pasados y presentes, que les ayuden a identificar el significado de las narraciones sobre el pasado, contextualizar y juzgar los diferentes hechos históricos, se encuentra bastante alejado de los conocimientos que se trabajan en la actualidad. Solo cuando estos contenidos se trasladen a las aulas y los alumnos puedan encontrar en ellos una utilidad real en su vida, podremos hablar de la historia como una materia que vela por la inclusión y el conocimientode cada uno de sus miembros y que puede favorecer el desarrollo de un sentimiento de pertenencia al contexto realmente justificado.

Estos déficits del currículo en cuanto a la significación de los contenidos históricos y las propias exigencias del contexto social requieren una mayor implicación del profesorado en la aplicación de estrategias que favorezcan la motivación para que los alumnos adquieran, como afirman Ortuño, Gómez y Ortiz (2012), unas habilidades que les permitan desenvolverse en la sociedad como ciudadanos responsables, participativos y en convivencia.

\subsection{Objetivo 2: percepción docente acerca de la metodología empleada}

Del final del objetivo anterior se desprende la idea de que las estrategias docentes utilizadas pueden suponer la diferencia entre la adquisición de aprendizajes perecederos y sin utilidad, basados en la capacidad memorística del alumnado, y la de aprendizajes verdaderamente significativos. Como afirman Mejía y Mejía:

Si las estrategias de los docentes en el aula de clase cambian, se podría esperar en los estudiantes actitudes que den cuenta realmente de lo que aprendieron, de cómo lo aprendieron y sobre todo para qué lo aprendieron, porque el pensamiento crítico contribuye a la formación de la autonomía personal, a enseñarle a los individuos la manera de potenciar sus habilidades intelectuales y a forjar una concepción del mundo razonada y comprendida (2015: 416).

Pero la utilización de estrategias alternativas por parte de los docentes va a depender, en gran medida, de su formación, no solo acerca de estas metodologías, sino y, sobre todo, en su consideración sobre lo que es la historia. Durante la recogida de información algunos de los docentes se mostraron de acuerdo con la selección de contenidos, afirmando que el currículum recoge los aspectos más importantes de la historia del país, aquellos contenidos que son imprescindibles para el alumnado y que los que no se enseñan es debido a la falta de tiempo o de relevancia de estos. Sin embargo, aquellos maestros más familiarizados con la historia o que han recibido una educación más amplia en esta materia son perfectamente conscientes de sus limitaciones y de los peligros que supone su enseñanza tradicional, unos peligros acrecentados especialmente, como propone Maldonado (2008), en la sociedad actual, en la que los docentes trabajan con grupos de estudiantes heterogéneos, que tienen diferentes estilos de aprendizaje, antecedentes étnicos y culturales y niveles de actividad.

Si hay algo en lo que todos los participantes de esta investigación han estado de acuerdo en todo momento ha sido en la idoneidad del uso de la metodología de Aprendizaje Basado en Proyectos para intentar guiar la enseñanza de la historia hacia la adquisición de un pensamiento crítico-metódico y que permita a los estudiantes comprender cómo conocemos el pasado y su uso para resolver interrogantes presentes, elementos clave del conocimiento histórico (Jenkins, 2008). La necesaria 
vinculación emocional con el pasado es lo que ayuda a dotar de sentido a la materia, así como sentirse parte de un discurso, lo que ha de redundar necesariamente en el sentimiento de inclusión de todos los participantes en el proyecto. Rodríguez, Vargas y Cortés, muestran las principales características y ventajas de esta metodología:

En la educación basada en proyectos, los docentes necesitan crear espacios para el aprendizaje, dando acceso a la información, soportando la enseñanza por la instrucción, modelamiento y guía a los estudiantes, para manejar de manera apropiada sus tareas, animarlos a utilizar procesos de aprendizaje metacognitivos, respetar los esfuerzos grupales e individuales, verificar el progreso, diagnosticar problemas, dar retroalimentación y evaluar los resultados generales (2010: 17).

En este sentido, Ortuño, Gómez y Ortiz (2012) proponen que el trabajo con este tipo de metodologías, que implican el uso de fuentes primarias o secundarias, estimula a los alumnos a continuar con la búsqueda de información de manera autónoma por medio de fuentes complementarias, sobre todo cuando los problemas estudiados tienen alguna relación con situaciones de su vida cotidiana. Consiguiendo, de este modo, el desarrollo de una gran independencia en los diferentes procesos de aprendizaje basada en la propia curiosidad por conocer y comprender los fenómenos que ocurren a su alrededor, cómo seleccionamos su estudio y cómo sabemos sobre ello. De este modo, como proponen Gómez, Ortuño y Molina (2014), al mismo tiempo que se enseñan valores de crítica y empatía no se deja de enseñar historia, puesto que lo que se consigue no es enseñar a los alumnos un contenido cerrado, sino cómo se consigue saber lo que sabemos sobre el pasado. En otras palabras, se consigue enseñar contenidos de historia y sobre la historia.

Por su parte, Maldonado (2008) considera que la aplicación de esta metodología proporciona experiencias de aprendizaje en las que el alumnado tiene la oportunidad de involucrarse en proyectos significativos que le permiten desarrollar de manera integral sus habilidades, actitudes y valores. Algo que también se ha podido comprobar durante la investigación, ya que todos los participantes estuvieron de acuerdo con los beneficios para las relaciones personales del empleo de esta metodología, por tanto y, de acuerdo con el mencionado Maldonado (2008), el trabajo de grupos colaborativos se convierte en un ingrediente esencial en todas las actividades de enseñanzaaprendizaje.

\subsection{Objetivo 3: percepción e interés de los alumnos por la materia}

Como pudimos comprobar con sus respuestas al cuestionario, el estudio de diferentes culturas suele servir al alumnado como motivación para seguir aprendiendo sobre la historia de su país. Sentirse identificados con ciertos contenidos y rasgos estudiados en la materia hace que muestren un mayor interés por conocer su procedencia y los cambios que han ido ocurriendo con el paso del tiempo. A pesar de esto, se ha comprobado una importante falta de conocimientos previos y vocabulario adecuado a los temas de historia, lo que se puede achacar de manera directa a la tradicional enseñanza basada en la memorización y que impide que los alumnos puedan otorgar cualquier utilidad real a sus contenidos e impidiendo la consecución de otro de los supuestos pilares fundamentales de la educación; la adquisición de aprendizajes significativos.

Navarro Barba (2006) afirma que para los niños dentro de los centros educativos ha dejado de ser algo anecdótico la presencia de la diversidad cultural, lo que les hacen incorporar las diferencias como algo positivo en la construcción de su identidad cultural. Es cierto, como se ha podido comprobar mediante el estudio de sus relaciones personales, que el alumnado no considera la diversidad cultural, étnica o cultural como un problema. Sin embargo, la existencia de narrativas maestras sobre la historia nacional puede suponer, en la actualidad, un obstáculo para el desarrollo de sentimientos de inclusión social y educativa y, por tanto, de referencia al entorno en el que se encuentran. Y el motivo de esto es que, en la selección tradicional de contendidos siguen sin darles voz, presencia o existencia a la diversidad y pluralidad entendida no como la enumeración de 
diferentes culturas sucesivas sin más conexión que la idea de conquista y superación, sino a los procesos e hibridaciones culturales. Se explica la historia de unas gentes que ni se parecen a ellos ni al origen de su cultura o a la presencia de la misma tanto en el presente como en el pasado, por lo que no consideran que esos contenidos sean suyos. El alumnado los estudia y los ve curiosos, pero no los hacen significativos. Por tanto, la historia, que puede dar voz amuchas gentes del pasado, no se usa para eso, sino para seguir hablando de las historias nacionales.

Aunque el cambio hacia la metodología de aprendizaje basado en proyectos pueda contribuir hacia un salto hacia la inclusión, por todos los beneficios educativos y sociales mencionados anteriormente, la Historia sigue sin posicionarse, de manera general, entre las materias preferidas por el alumnado. La falta de representación, es decir, el estudio de una historia considerada como ajena, hace que el alumnado pierda el interés por la misma y, aunque disfruten con su metodología, no consiguen disfrutar con sus contenidos. En este sentido, el motivo radica en una concepción epistemológica de la historia que hace que no se le vea más sentido o potencial que el descubrimiento del pasado en lugar de la interpretación del mismo o la solución a los interrogantes actuales. Dicho de otro modo, una metodología activa no conlleva ningún beneficio si no hay un acompañamiento que suponga que el conocimiento histórico también es un conocimiento científico, con lo que supone de acercamiento el método, finalidad y dificultades de generación dicho conocimiento. Lo que nos recuerda al estudio llevado a cabo por Von Borries (2006), en el que el alumnado alemán de origen turco, a pesar de encontrarse realizando una de las actividades complementarias de las que más suele disfrutar el alumnado, una salida escolar, no mostraban ningún interés por la misma, excusando su despiste en que la historia de la Alemania nazi no les definía, pues su origen era diferente. Mostraban, por tanto, una falta total de empatía ante esos acontecimientos. Esta es la situación que se está viviendo en nuestros centros escolares en los que, por culpa de los contenidos históricos que se intentan inculcar, los alumnos han perdido el interés por conocer la Historia.

\section{Conclusión}

Para dar respuesta al problema de investigación planteado al comienzo del estudio debemos tener en cuenta todos los aspectos estudiados anteriormente, así como el resto de las variables que influyen en la adquisición, o no, de un sentimiento de pertenencia al entorno por parte del alumnado. Pues, como pudimos comprobar en el análisis de la información recogida durante la investigación, el profesorado achaca, por un lado, los principales conflictos que pueden surgir entre el alumnado a los valores transmitidos desde las familias que, de manera consciente o inconsciente, fomentan el uso de argumentos racistas contra sus compañeros, y la falta de adaptación al centro al escaso conocimiento del lenguaje por parte del alumnado que, aunque nacido en España, tiene su origen en un país de habla distinta al castellano, siendo la de su país de origen la que hablan de manera habitual fuera del centro escolar.

Escudero (2005) propone que la exclusión es el resultado de la confluencia e interacción de diversos elementos, estructuras y dinámicas diferentes, y que son situadas, además, en diversos niveles como pueden ser los aspectos personales y sociales de los estudiantes, las características familiares, la influencia del grupo de iguales, las características de la comunidad de residencia, el entorno escolar y las políticas sociales y educativas, así como la propia formación del profesorado. Por todo esto, no somos tan temerarios de culpar a una materia de que el alumnado en nuestro país se pueda sentir en situación de exclusión. A pesar de esto, debemos resaltar que la influencia de la enseñanza de la materia de Historia es innegable para intentar conseguir generar un sentimiento de identidad colectiva, de sobra ya comentado por la literatura e implícitos en el tratamiento curricular.

Por ello y, en primer lugar, debemos tener en cuenta la propuesta de Prats y Valls (2011), en la que afirman el enorme impacto social y la influencia que supone el área de historia tanto en la educación como en el conjunto de toda la sociedad. Por lo que resulta necesario conocer perfectamente a nuestro alumnado y someter a la educación a las renovaciones necesarias que le 
permitan adaptarse a estas características, procurando de este modo que se convierta en un medio que facilite la inclusión, algo que no está consiguiendo en la actualidad debido al tratamiento insuficiente de la diversidad cultural y a la compartimentación y descontextualización de sus contenidos, entre otros aspectos.

Sin embargo, encontramos un aspecto muy positivo durante la investigación al comprobar la disposición para el cambio en el profesorado que, a pesar de las grandes limitaciones curriculares, se esfuerza por utilizar metodologías que faciliten la inclusión como el aprendizaje basado en proyectos, mostrado por ser el utilizado en el centro de referencia, que brinda al alumnado la posibilidad de seleccionar sus propios contenidos, de acercarse al desarrollo del pensamiento histórico crítico y, además, por producir beneficios en el desarrollo de su autonomía y de sus relaciones personales. Molina, Miralles, Deusdad y Alfageme (2017) afirman en este sentido que el primer paso no es tanto modificar el currículo disciplinar, academicista y culturalista actual, sino llevar acabo unas prácticas docentes más activas y que tengan en cuenta el interés y las características del alumnado, así como una formación específica sobre la fundamentación epistemológica de la disciplina.

Por tanto y, aunque en la actualidad la respuesta al problema de investigación sea negativa, el dato anterior nos permite empezar a pensar que, remitiéndonos a la cita utilizada en el marco conceptual en la que Bauman (1999) afirmaba que nos encontramos en una época de pluralidad cultural y no de pluralidad de culturas, la enseñanza de la historia puede pasar de una orientación basada en la diversidad cultural, a la cultura de la diversidad (Leiva, 2011).

\section{Referencias}

Aranguren, C. (2006). Ciudadanía intercultural, enseñanza de la historia y exclusión social. En Encuentro de Latinoamericanistas Españoles: Viejas y nuevas alianzas entre América Latina y España (pp. 196-204). Madrid: Consejo Español de Estudios Iberoamericanos.

Ávila-Fuenmayor, F. (2006). El concepto de poder en Michel Foucault. Telos, 2, 215-234.

Bauman, Z., (1999). La cultura como praxis. Barcelona: Editorial Paidós Ibérica.

Borries, B., Von. (2006). ¡Qué nos importa vuestra historia! Enseñanza de historia en una sociedad de inmigración, Íber, 47.

Carretero, M. (2000). Cambio conceptual y enseñanza de la historia. Tarbiya: Revista de investigación e innovación educativa, 26, 73-82.

Carretero, M., Castorina, J. A., Sarti, M., van Alphen, F., Barreiro, A. (2013). La construcción del conocimiento histórico.Propuesta educativa,39.

Carretero, M. y Kriger, M. (2004). ¿Forjar patriotas o educar cosmopolitas? El pasado y el presente de la historia escolar en un mundo global. En M. Carretero y J. F. Voss (coord.) Aprender y pensar la Historia. Buenos Aires: Amorrortu.

Castells, M. (2005). The Network Society: From Knowledge to Policy. En M. Castells y G. Cardoso (eds.), The Network Society: From Knowledge to Policy (pp. 3-21). Washington: John Hopkins University Press.

Cuesta, R. (2000). Usos y abusos de la educación histórica. Didáctica de las Ciencias Experimentales y Sociales, 14, 23-31.

Escudero, J. (2005). Fracaso escolar, exclusión educativa: ¿De qué se excluye y cómo? Profesorado, Revista de Currículum y Formación de Profesorado, 9.

Fontana, J. (2006). ¿Qué historia para el siglo XXI? Analecta: Revista de Humanidades, 1, 1-12.

Giroux, H. (1994). Hacia una pedagogía de la política de la diferencia. En H. Giroux, y R. Flecha (coord.). Igualdad educativa y diferencia cultural (pp. 76-94). Barcelona: El Roure, S.A.

Gómez, C. J., Ortuño, J. y Molina, S. (2014). Aprender a pensar históricamente. Retos para la historia en el siglo XXI. Revista Tempo e Argumento, 11, 5-27.

Jenkins, K. (2008). Repensar la historia. Madrid: Siglo XXI. 
Leiva, J. J. (2011). La educación intercultural: un compromiso educativo para construir una escuela sin exclusiones. Revista Iberoamericana de Educación, 56.

Maldonado, M. (2008). Aprendizaje basado en proyectos colaborativos. Una experiencia en educación superior. Laurus, Revista de educación, 28, 158-180.

Martínez Carazo, P. C. (2006). El método de estudio de caso: estrategia metodológica de la investigación científica. Pensamiento y gestión, 20, 165-193.

Mejía. L. y Mejía, A. L. (2015). Relaciones entre pensamiento histórico y pensamiento crítico en la enseñanza de las ciencias sociales en estudiantes de educación básica secundaria, RIIEP, 2, 413-436.

Molina, S., Miralles, P., Deusdad, B. y Alfageme, M. B. (2017). Enseñanza de la historia, creación de identidades y prácticas docentes. Profesorado, Revista de currículum y formación del profesorado,21 (2), 331-354.

Molina, S., Miralles, P. y Ortuño, J. (2013). Concepciones de los futuros maestros de Educación Primaria sobre formación cívica y ciudadana. Educatio Siglo XXI, 31, 105-126.

Navarro Barba, J. (2006). La escolarización de niños inmigrantes en la Región de Murcia: diez años, diez razones para pensar en sus retos, mitos y realidades. Anales de Historia Contemporánea, 22, 136-150.

Okuda, M. y Gómez-Restrepo, C. (2005). Métodos en investigación cualitativa: triangulación. Revista colombiana de psiquiatría, 34 (1), 118-124.

Ortuño, J., Gómez, C. J. y Ortiz, E. (2012). La evaluación de la competencia educativa social y ciudadana desde la didáctica de las ciencias sociales. Un estado de la cuestión. Didáctica de las Ciencias Experimentales y Sociales, 26, 53-72.

Pagès, J. (2002). Aprender a enseñar historia y ciencias sociales: el currículo y la didáctica de las ciencias sociales. Pensamiento Educativo, 30, 255-269.

Pérez Garzón, S. J. (2002). Usos y abusos de la historia. Gerónimo de Uztariz, 17-18, 11-24.

Prats, J. y Valls, R. (2011). La didáctica de la Historia en España: estado reciente de la cuestión. Didáctica de las Ciencias Experimentales y Sociales, 25, 17-35.

Rodríguez-Sandoval, E., Vargas-Solano, E. M. y Luna Cortés, J. (2010). Evaluación de la estrategia "aprendizaje basado en proyectos". Educación y Educadores, 1, 13-25.

Silva, D. F. (2011). Alteridad y conflicto: la tarea hermenéutica de la democracia. Polisemia, 11, 5061.

CÓMO CITAR ESTE ARTÍCULO

Cardoso, N., Molina, S. y Ortuño, J. (2020). Identificación del alumnado de Educación Primaria con las narrativas históricas locales y nacionales. Estudio de caso en un contexto multicultural. Didáctica de las ciencias experimentales y sociales, 38, 17-38. DOI: 10.7203/DCES.38.13491 


\section{ANEXOS}

\section{ANEXO 1. Rúbrica de evaluación mediante observación directa al alumnado.}

Rúbrica de escala Likert compuesta por 17 ítems, cuyo objetivo es recoger la información sobre el alumnado observada en el aula durante la realización del proyecto. Los ítems están divididos en dos ámbitos:

1. Observar la actitud del alumnado frente al tema y la tarea (ítems 1-9).

2. Observar la actitud del alumnado con sus compañeros (ítems 10-17).

\begin{tabular}{|c|c|c|c|c|c|}
\hline ÍTEMS & $\begin{array}{l}\stackrel{\Xi}{\Xi} \\
\bar{\Xi}\end{array}$ & 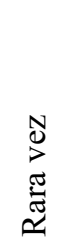 & 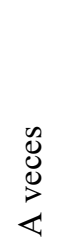 & 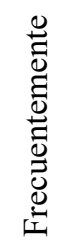 & 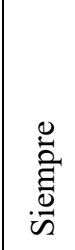 \\
\hline 1. Conoce algún dato sobre el tema antes de comenzar. & & & & & \\
\hline 2. Muestra interés inicial por el tema que debe trabajar. & & & & & \\
\hline $\begin{array}{l}\text { 3. Muestra interés por el tema que otros grupos deben } \\
\text { desarrollar. }\end{array}$ & & & & & \\
\hline 4. Mantiene una actitud positiva durante todo el proyecto. & & & & & \\
\hline 5. Participa activamente en la búsqueda de información. & & & & & \\
\hline 6. Permanece centrado en la tarea durante toda la sesión. & & & & & \\
\hline $\begin{array}{l}\text { 7. Pregunta dudas sobre los contenidos, busca apoyo o } \\
\text { explicaciones complementarias por parte del maestro/a. }\end{array}$ & & & & & \\
\hline $\begin{array}{l}\text { 8. Selecciona y genera los contenidos más relevantes para } \\
\text { su exposición. }\end{array}$ & & & & & \\
\hline $\begin{array}{l}\text { 9. Añade información complementaria de su interés que } \\
\text { considera que puede interesar a sus compañeros/as. }\end{array}$ & & & & & \\
\hline $\begin{array}{l}\text { 10. Mantiene una actitud positiva con los miembros de su } \\
\text { grupo. }\end{array}$ & & & & & \\
\hline 11. Es un líder para su equipo. & & & & & \\
\hline 12. Contribuye en el trabajo al mismo nivel que los demás. & & & & & \\
\hline $\begin{array}{l}\text { 13. Escucha las aportaciones de los demás con respeto y } \\
\text { tolerancia ante sus opiniones. }\end{array}$ & & & & & \\
\hline $\begin{array}{l}\text { 14. Genera debates que sirven para enriquecer los } \\
\text { contenidos del proyecto. }\end{array}$ & & & & & \\
\hline 15. Valora a todos sus compañeros/as por igual. & & & & & \\
\hline $\begin{array}{l}\text { 16. Contribuye a crear un clima de trabajo relajado y } \\
\text { responsable. }\end{array}$ & & & & & \\
\hline $\begin{array}{l}\text { 17. Intenta solucionar los problemas que surgen durante el } \\
\text { desarrollo del proyecto. }\end{array}$ & & & & & \\
\hline
\end{tabular}


ANEXO 2. Actividad alumnado para conocer su asignatura favorita.

Nombre:

Enumera, por orden de preferencia, tus asignaturas favoritas de las propuestas a continuación. Al lado de cada asignatura escribe un adjetivo con el que quieras definirla.

LENGUA

INGLÉS

EDUCACIÓN FÍSICA

MATEMÁTICAS

HISTORIA

VALORES SOCIALES Y CÍVICOS / RELIGIÓN

\begin{tabular}{|l|l|}
\hline \multicolumn{1}{|c|}{ ASIGNATURA } & ADJETIVO \\
\hline 1. & \\
\hline 2. & \\
\hline 3. & \\
\hline 4. & \\
\hline 5. & \\
\hline 6. & \\
\hline
\end{tabular}

Mi asignatura favorita es:

Porque: 


\section{ANEXO 3. Cuestionario alumnado}

Cuestionario diseñado para conocer la percepción del alumnado de Educación Primaria con respecto al clima escolar, el desarrollo de valores de tolerancia y respeto y sus conocimientos acerca de la historia.

\begin{tabular}{|c|c|c|c|c|c|}
\hline & 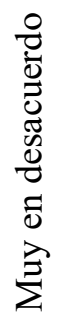 & 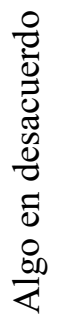 & 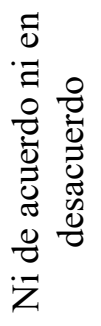 & 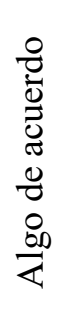 & 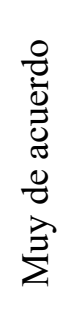 \\
\hline $\begin{array}{l}\text { 1. Los contenidos que aprendo en historia son de mi } \\
\text { interés. }\end{array}$ & & & & & \\
\hline $\begin{array}{l}\text { 2. Recuerdo los temas que he trabajado en historia con el } \\
\text { paso del tiempo. }\end{array}$ & & & & & \\
\hline $\begin{array}{l}\text { 3. Considero que lo que aprendo en historia me puede } \\
\text { servir para mi vida. }\end{array}$ & & & & & \\
\hline $\begin{array}{l}\text { 4. El modo de trabajar la historia por medio de proyectos } \\
\text { me resulta divertido. }\end{array}$ & & & & & \\
\hline $\begin{array}{l}\text { 5. Me gusta crear junto con mis compañeros/as los } \\
\text { contenidos que aprendemos en historia. }\end{array}$ & & & & & \\
\hline $\begin{array}{l}\text { 6. Investigar de manera autónoma me ayuda a aprender } \\
\text { más historia. }\end{array}$ & & & & & \\
\hline $\begin{array}{l}\text { 7. Aprender historia me ayuda a comprender mejor el } \\
\text { mundo en el que vivo. }\end{array}$ & & & & & \\
\hline $\begin{array}{l}\text { 8. Creo que los hechos del pasado han tenido } \\
\text { consecuencias en el presente. }\end{array}$ & & & & & \\
\hline $\begin{array}{l}\text { 9. Aprendo historia sobre otras culturas diferentes a la } \\
\text { española. }\end{array}$ & & & & & \\
\hline $\begin{array}{l}\text { 10. Me siento identificado con las culturas que se trabajan } \\
\text { en la escuela. }\end{array}$ & & & & & \\
\hline $\begin{array}{l}\text { 11. La historia me ayuda a desarrollar valores de respeto } \\
\text { y tolerancia hacia otras culturas. }\end{array}$ & & & & & \\
\hline $\begin{array}{l}\text { 12. Aprender historia no tiene nada que ver con el } \\
\text { presente. }\end{array}$ & & & & & \\
\hline $\begin{array}{l}\text { 13. La manera de evaluar la historia me permite expresar } \\
\text { mi opinión sobre los hechos aprendidos. }\end{array}$ & & & & & \\
\hline $\begin{array}{l}\text { 14. Aprender historia solo significa conocer los hechos } \\
\text { más importantes del pasado. }\end{array}$ & & & & & \\
\hline $\begin{array}{l}\text { 15. La maestra siempre está dispuesta a ayudarnos a } \\
\text { encontrar la información más adecuada. }\end{array}$ & & & & & \\
\hline $\begin{array}{l}\text { 16. La historia que aprendo en la escuela es la misma que } \\
\text { me enseña mi familia/amigos fuera de ella. }\end{array}$ & & & & & \\
\hline $\begin{array}{l}\text { 17. Obtenemos la información desde varias fuentes } \\
\text { (consultando diferentes páginas web, libros, etc.) }\end{array}$ & & & & & \\
\hline $\begin{array}{l}\text { 18. Aprender historia sobre mi propia cultura en la escuela } \\
\text { hace que me motive para seguir estudiando. }\end{array}$ & & & & & \\
\hline
\end{tabular}




\section{ANEXO 4. Entrevista semi-estructurada profesorado}

Se trata de una entrevista semi-estructurada dirigida a los maestros y maestras de Educación Primaria de un centro de titularidad pública de Cartagena.

La entrevista consta de 10 preguntas y su objetivo principal es conocer la percepción de los docentes acerca de su trabajo con alumnos/as en un contexto de diversidad cultural, centrándonos en su opinión acerca de los contenidos históricos impartidos y sus objetivos en la escuela. Podemos definir dos grupos de preguntas según su temática:

- Preguntas relacionadas con la trayectoria docente de los entrevistados y sus percepciones sobre el centro. (1-5).

- Preguntas relacionadas con la enseñanza de la historia (6-10).

1. ¿Por qué decidiste ser maestro/a de Educación Primaria?

2. ¿Qué te supone personalmente trabajar en un centro de características y necesidades tan diversas?

3. ¿Qué relación mantienen los alumnos y alumnas entre sí?, ¿crees que existe algún tipo de discriminación en el aula?

4. ¿Qué metodología docente utilizas normalmente en tus clases?, ¿crees que la metodología influye en sus relaciones?

5. ¿Qué materias crees que gustan más a los alumnos/as?

6. ¿Qué temas de la historia interesan más a los alumnos/as?

7. ¿Por qué crees que es importante que los alumnos/as aprendan historia?

8. ¿Crees que la historia que se enseña es la adecuada?

9. ¿Cuál crees que es el objetivo que persigue la enseñanza de la historia desde el currículo?, ¿qué valores pretenden inculcar sus contenidos?

10. ¿Cuál es tu objetivo principal al enseñar historia?, ¿crees que mediante su enseñanza se pueden desarrollar valores que favorezcan la inclusión de todos los alumnos/as? 